Pacific Journal of Mathematic 


\title{
A MEAN VALUE THEOREM FOR BINARY DIGETS
}

\author{
Alan H. Stein and Ivan E. Stux
}

This paper continues the investigation of the dyadically additive function $\alpha$ defined by $\alpha(n)=$ the number of 1 's in the binary expansion of $n$.

Previously, Bellman and Shapiro (cf. "On a problem in additive number theory." Annals of Mathematics, 49 (1948) 333-340) showed that $\sum_{k=1}^{x} \alpha(k) \sim x \log x / 2 \log 2$. They then considered the iterates of $\alpha$ defined by $\alpha_{q}=\alpha_{q-1} \circ \alpha$ and observed that $A_{r}(x)=\sum_{k=1}^{x} \alpha_{r}(k)$ is not asymptotic to any elementary function for $r \geqq 2$.

In this paper the function $A_{2}(x)$ will be examined more closely. Defining $c(x)$ by $A_{2}(x)=c(x) x \log \log x / 2 \log 2$, we will prove the following theorems.

THEOREM 1. As $x$ ranges over the positive integers, $c(x)$ ranges densely over $[1 / 2,3 / 2]$. Furthermore, given any $c \in[1 / 2,3 / 2]$, there is an explicit way to construct a sequence of integers $x$ for which $c(x) \rightarrow c$ as $x \rightarrow \infty$.

THEOREM 2.

$$
1 / 2+O(\log \log \log x / \log \log x) \leqq c(x)
$$

$$
\leqq 3 / 2+O(\log \log \log x / \log \log x) .
$$

THEOREM 3.

$$
\liminf c(x)=1 / 2, \quad \limsup c(x)=3 / 2
$$

Note. Theorem 3 is an immediate consequence of Theorems 1 and 2.

2. The proof of Theorem 1 is obtained by considering a special set of integers.

Let $\mathcal{M}=\left\{x: x=2^{M}-1, M\right.$ even, $M / 2=\sum_{i=1}^{r} 2^{a_{1}}-1, a_{1}>a_{2}>\cdots>$ $a_{r}$ positive integers, and $\left.a_{r} / a_{1} \geqq 1 / 2+\log \log \log x / \log \log x\right\}$.

LEMMA 1. If $x \in \mathcal{M}$, then 


$$
A_{2}(x)=\left(r+\frac{a_{r}}{2}+O(1)\right) x
$$

Proof. If $x \in \mathcal{M}$, then (cf. [1])

$$
A_{2}(x)=\sum_{k<2^{M}} \alpha(\alpha(k))=\sum_{n \leq M}\left(\begin{array}{c}
M \\
n
\end{array}\right) \alpha(n)
$$

We can then write

$$
A_{2}(x)=\sum_{1}\left(\begin{array}{l}
M \\
n
\end{array}\right) \alpha(n)+\sum_{2}\left(\begin{array}{l}
M \\
n
\end{array}\right) \alpha(n)
$$

where $\Sigma_{1}$ is the sum over $\left\{n:|M / 2-n|<2 a_{r}\right\}$ and $\Sigma_{2}$ is the sum over $\left\{n:|M / 2-n| \geqq 2 a_{r}\right\}$.

Chebyshev's inequality yields

$$
\sum_{2}\left(\begin{array}{l}
M \\
n
\end{array}\right) \alpha(n) \ll 2^{M} M \cdot 2^{-2 a_{r}} \log M
$$

$\ll 2^{M} \cdot M \cdot 2^{-a_{1}(1+2 \log \log \log x / \log \log x)} \log M$

which implies

$$
\sum_{2}\left(\begin{array}{c}
M \\
n
\end{array}\right) \alpha(n)=O(x)
$$

(Here and further on, inequalities such as $M=O(\log x), a_{1}=$ $O(\log M), \alpha(n)=O(\log n)$ and $r=O(\log M)$ will be used without comment).

We will use the symmetry of the binomial coefficients to estimate $\Sigma_{1}$.

$$
\sum_{1}\left(\begin{array}{l}
M \\
n
\end{array}\right) \alpha(n)=\frac{1}{2} \sum_{0 \neq|t| i \mid<2_{r}}\left(\begin{array}{c}
M \\
M / 2+t
\end{array}\right)\left\{\alpha\left(\frac{M}{2}-t\right)+\alpha\left(\frac{M}{2}+t\right)\right\}
$$

$$
+\left(\begin{array}{c}
M \\
M / 2
\end{array}\right) \alpha(M / 2)
$$

Writing $t=\sum_{j=1}^{w} 2^{b,}$, we obtain

$$
\alpha\left(\frac{M}{2}+t\right)=\alpha\left(\sum_{i=1}^{r} 2^{a_{t}}+\sum_{j=1}^{w} 2^{b_{l}}-1\right)=r+w-1+b_{w}
$$


and

(2.8)

$$
\alpha\left(\frac{M}{2}-t\right)=\alpha\left(\sum_{t=1}^{r} 2^{a_{i}}-1-\sum_{j=1}^{w} 2^{b_{j}}\right)=r-1+a_{r}-w
$$

so that

$$
\alpha\left(\frac{M}{2}+t\right)+\alpha\left(\frac{M}{2}-t\right)=2 r-2+a_{r}+b_{w}
$$

We can now rewrite (2.6), obtaining

$$
\sum_{1}\left(\begin{array}{c}
M \\
n
\end{array}\right) \alpha(n)=\sum_{0<|r|<2^{a r}}\left(\begin{array}{c}
M \\
M / 2+1
\end{array}\right)\left(r-1+\frac{a_{r}}{2}+\frac{b_{w}}{2}\right)+\left(\begin{array}{c}
M \\
M / 2
\end{array}\right) a_{r}
$$

Chebyshev's inequality implies that

$$
\sum_{|t| \geq 2^{a_{r}}}\left(\begin{array}{c}
M \\
M / 2+t
\end{array}\right)\left(r+\frac{a_{r}}{2}\right)=O(x)
$$

as in the analysis of $\Sigma_{2}$. Since

$$
\left(\begin{array}{c}
M \\
M / 2
\end{array}\right)\left(r+a_{r} / 2\right)=O(x \log \log x / \sqrt{\log x})=O(x)
$$

we obtain

$$
\sum_{|r|<2^{a_{r}}}\left(\begin{array}{c}
M \\
M / 2+t
\end{array}\right)\left(r+\frac{a_{r}}{2}\right)=2^{M}\left(r+\frac{a_{r}}{2}\right)+O(x)=\left(r+\frac{a_{r}}{2}\right)+O(x)
$$

Thus it remains only to show that each remaining term is $O(x)$. We have already seen that

$$
\left(\begin{array}{c}
M \\
M / 2
\end{array}\right) a_{r} / 2=O(x)
$$

and easily obtain

$$
\sum_{|t|<2^{a_{r}}}\left(\begin{array}{c}
M \\
M / 2+t
\end{array}\right)(-1)=O\left(2^{M}\right)=O(x)
$$

We estimate the remaining term by observing that $b_{w}=b_{w}(t)$ is the largest exponent such that $2^{b_{w}} \mid t$. Thus we can write 
(2.14)

$$
\begin{aligned}
\sum_{0<|t|<2^{a_{r}}}\left(\begin{array}{c}
M \\
M / 2+t
\end{array}\right) b_{w} & \leqq \sum_{\substack{h, t \\
2^{\prime} \mid t}}\left(\begin{array}{c}
M \\
M / 2+t
\end{array}\right) \leqq \sum_{t \geq 0} \sum_{q>0}\left(\begin{array}{c}
M \\
M / 2+2^{\llcorner q}
\end{array}\right) \\
& \leqq \sum_{t \geq 0} \frac{1}{2^{t}} \sum_{q}\left(\begin{array}{c}
M \\
q
\end{array}\right)=O(x) .
\end{aligned}
$$

This completes the proof of Lemma 1 .

Lemma 1 implies that

$$
c(x)=\frac{r+a_{r} / 2}{\log \log x / 2 \log 2}+o(1)
$$

Since $a_{1}=\log \log x / 2 \log 2+O(1)$, we obtain

$$
c(x)=\frac{r+a_{r} / 2}{a_{1}}+o(1)
$$

We now complete the proof of Theorem 1 by showing that if $\epsilon>0$ then there exist arbitrarily large $q$ such that if $(1 / 2+\epsilon) q<z<(3 / 2-\epsilon) q$ is an integer, then there exists $x \in \mathcal{M}$ such that $a_{1}=q$ and $r+a_{r} / 2=z$.

Suppose we choose $(1 / 2+\epsilon) q-4 \leqq s<(1 / 2+\epsilon) q-2, s$ even. As $t$ takes on all possible integer values between 2 and $q-s, t+s / 2$ certainly takes on all integer values between $(1 / 2+\epsilon) q$ and $(3 / 2-\epsilon) q$.

If $q$ is large enough, it is certainly possible to find $x \in \mathcal{M}$ such that $a_{1}=q, r=t$ and $a_{r}=s$, completing the proof of Theorem 1 .

3. We carry out the proof of Theorem 2 in a series of steps.

Let $M^{1}=\left\{x: x=2^{M}-1, M\right.$ even, $M / 2=\sum_{i=1}^{r} 2^{a_{i}}-1, a_{1}>a_{2}>\cdots>$ $a_{r}$ integers and $\left.a_{r} / a_{1} \geqq(1 / 2) \log \log \log x /(\log \log x+\log \log 2)\right\}$. of $\mathscr{M}^{1}$.

We begin by proving the conclusion of Theorem 2 holds for element

LeMma 2. If $x \in M^{1}$ then

$$
\frac{1}{2}+O\left(\frac{\log \log \log x}{\log \log x}\right)<c(x)<\frac{3}{2}+O\left(\frac{\log \log \log x}{\log \log x}\right)
$$

Proof. We begin as in Lemma 1, writing

$$
A_{2}(x)=\sum_{1}\left(\begin{array}{l}
M \\
n
\end{array}\right) \alpha(n)+\sum_{2}\left(\begin{array}{c}
M \\
n
\end{array}\right) \alpha(n)
$$


except where $\Sigma_{1}$ is the sum over $\left\{n:|M / 2-n|<2^{\left(\frac{1}{2}+\epsilon\right) a_{1}}\right\}$ and $\Sigma_{2}$ is the sum over $\left\{n:|M / 2-n| \geqq 2^{(1 / 2+\epsilon) a_{1}}\right\}$, where $\epsilon=\epsilon(x)=$ $\log \log \log x /(\log \log x+\log \log 2)$.

The second term can be estimated as the corresponding term was in Lemma 1, yielding

$$
\sum_{2}\left(\begin{array}{c}
M \\
n
\end{array}\right) \alpha(n)=O(x)
$$

We estimate the first sum by considering two cases.

Case 1. $a_{r} \geqq(1 / 2+\epsilon) a_{1}$. We can treat this case as we treated Lemma 1 , obtaining $\Sigma_{1}\left(\begin{array}{c}M \\ n\end{array}\right) \alpha(n)=\left(r+a_{r} / 2\right) x+O(x)$ and hence

$$
A_{2}(x)=\left(r+a_{r} / 2\right) x+O(x)
$$

Since $0 \leqq r \leqq a_{1}-a_{r}+1$, we obtain $a_{r} / 2 \leqq r+a_{r} / 2 \leqq a_{1}-a_{r} / 2+1$. Since $(1 / 2+\epsilon) a_{1} \leqq a_{r} \leqq a_{1}$, we obtain

$$
\left(\frac{1}{4}+\frac{\epsilon}{2}\right) a_{1} \leqq r+a_{r} / 2 \leqq\left(\frac{3}{4}-\frac{\epsilon}{2}\right) a_{1}+1
$$

But $a_{1}=(\log \log x / \log 2)+O(1)$, so

$$
\left(\frac{1}{4}+\frac{\epsilon}{2}\right) \frac{\log \log x}{\log 2}+O(1) \leqq r+a_{r} / 2 \leqq\left(\frac{3}{4}-\frac{\epsilon}{2}\right) \frac{\log \log x}{\log 2}+O(1)
$$

which implies

$$
\frac{1}{4} \frac{\log \log x}{\log 2}+O(\log \log \log x) \leqq r+a_{r} / 2 \leqq \frac{3}{4} \frac{\log \log x}{\log 2}+O(\log \log \log x) .
$$

Thus

$$
\begin{gathered}
\left(\frac{1}{2}+O\left(\frac{\log \log \log x}{\log \log x}\right)\right)\left(\frac{x \log \log x}{2 \log 2}\right) \leqq A_{2}(x) \\
\leqq\left(\frac{3}{2}+O\left(\frac{\log \log \log x}{\log \log x}\right)\right)\left(\frac{x \log \log x}{2 \log 2}\right)
\end{gathered}
$$

which proves Lemma 2 for this case. 
Case 2. $(1 / 2-\epsilon) a_{1}<a_{r}<(1 / 2+\epsilon) a_{1}$. As in Lemma 1, we write

$$
\sum_{1}\left(\begin{array}{c}
M \\
n
\end{array}\right) \alpha(n)=\frac{1}{2} \sum_{0 \neq t<2^{(1 / 2+\epsilon) a_{1}}}\left(\begin{array}{c}
M \\
M / 2+t
\end{array}\right)\left\{\alpha\left(\frac{M}{2}-t\right)+\alpha\left(\frac{M}{2}+t\right)\right\}
$$

$$
+\left(\begin{array}{c}
M \\
M / 2
\end{array}\right) \alpha\left(\frac{M}{2}\right)
$$

Here, however, an overlap of the nonzero digits in the binary representations of $t$ and $M / 2+1$ forces us to use the subadditive properties of $\alpha$. Writing $M / 2$ and $t$ as before, we obtain

$$
\frac{M}{2}+t=2^{a_{1}}+\cdots+2^{a_{r}}+2^{b_{1}}+\cdots+2^{b_{w}}-1
$$

$$
\frac{M}{2}-t=2^{a_{1}}+\cdots+2^{a_{r}}-2^{b_{1}}-\cdots-2^{b_{w}}-1
$$

The subadditivity of $\alpha$ implies $\alpha(M / 2+t) \leqq \alpha(M / 2+1)+\alpha(t-1)$ so that

$$
\alpha\left(\frac{M}{2}+t\right) \leqq r+w+b_{w}
$$

Also, $\alpha(M / 2+t)$ is at least $\alpha(t)$ minus the overlap between the binary expansions of $M / 2$ and $t$, so that

$$
\alpha\left(\frac{M}{2}+t\right) \geqq w-2 \epsilon a_{1} .
$$

Since $\alpha(M / 2-t)$ is no greater than the number of places available, less $\alpha(t)$, plus the overlap, we obtain

$$
\alpha\left(\frac{M}{2}-t\right) \leqq a_{1}+1-w+2 \epsilon a_{1}
$$

Also, $\alpha(M / 2-t)$ must be at least the number of 1 's that $M / 2$ ends with less $\alpha(t)$, so that

$$
\alpha\left(\frac{M}{2}-t\right) \geqq a_{r}-w
$$

Combining (3.11)-(3.14) we obtain 
(3.15) $a_{r}-2 \epsilon a_{1} \leqq \alpha\left(\frac{M}{2}+t\right)+\alpha\left(\frac{M}{2}-t\right) \leqq a_{1}+r+b_{w}+2 \epsilon a_{1}+1$.

Since $a_{r}>(1 / 2-\epsilon) a_{1}$ and $r \leqq a_{1}-a_{r}+1<a_{1}-(1 / 2-\epsilon) a_{1}+1=$ $(1 / 2+\epsilon) a_{1}+1$ we obtain

$$
\left(\frac{1}{2}-3 \epsilon\right) a_{1} \leqq \alpha\left(\frac{M}{2}+t\right)+\alpha\left(\frac{M}{2}-t\right) \leqq\left(\frac{3}{2}+3 \epsilon\right) a_{1}+b_{w}+1
$$

Plugging the first inequality of (3.16) into (3.9) yields

$$
\sum_{1}\left(\begin{array}{c}
M \\
n
\end{array}\right) \alpha(n) \geqq \frac{1}{2}\left(\frac{1}{2}-3 \epsilon\right) a_{1} \sum_{0 \neq t<2^{(1 / 2+\infty) a_{1}}}\left(\begin{array}{c}
M \\
M / 2+t
\end{array}\right)+\left(\begin{array}{c}
M \\
M / 2
\end{array}\right) \alpha\left(\frac{M}{2}\right) .
$$

Chebyshev's inequality yields

$$
\sum_{0 \neq t<2^{(1 / 2+o) a_{1}}}\left(\begin{array}{c}
M \\
M / 2+t
\end{array}\right)=x+O\left(\frac{x}{2^{2 \varepsilon a_{1}}}\right)
$$

which implies

$$
\sum_{1}\left(\begin{array}{l}
M \\
n
\end{array}\right) \alpha(n) \geqq \frac{1}{4} a_{1} x-\frac{3}{2} \epsilon a_{1} x+O(x)
$$

Recalling $\left.a_{1}=(\log \log x) / \log 2\right)+O(1)$ and combining (3.17) with (3.3) yields

$$
A_{2}(x) \geqq \frac{1}{2} \frac{x \log \log x}{2 \log 2}+O(x \log \log \log x)
$$

which implies

$$
c(x) \geqq \frac{1}{2}+O\left(\frac{\log \log \log x}{\log \log x}\right)
$$

Plugging the second inequality of (3.16) into (3.9) yields

$$
\begin{aligned}
\sum_{1}\left(\begin{array}{c}
M \\
n
\end{array}\right) \alpha(n) \leqq & \frac{1}{2}\left(\frac{3}{2}+3 \epsilon\right) a_{1} \sum_{0 \neq t<2^{(1 / 2+e) a_{1}}}\left(\begin{array}{c}
M \\
M / 2+t
\end{array}\right) \\
& +\frac{1}{2} \sum_{0<|t|<2^{(1 / 2+o) a_{1}}}\left(\begin{array}{c}
M \\
M / 2+t
\end{array}\right)\left(b_{w}+1\right) \\
& +\left(\begin{array}{c}
M \\
M / 2
\end{array}\right) \alpha\left(\frac{M}{2}\right) .
\end{aligned}
$$


As in (2.14) we see that

$$
\sum_{0<|t|<2^{(1 / 2+\infty) a_{1}}}\left(\begin{array}{c}
M \\
M / 2+t
\end{array}\right)\left(b_{w}+1\right)=O(x)
$$

to obtain

$$
\sum_{1}\left(\begin{array}{c}
M \\
n
\end{array}\right) \alpha(n) \leqq \frac{3}{4} a_{1} x+\frac{3}{2} \epsilon a_{1} x+O(x)
$$

Repeating the reasoning of (3.17)-(3.19) yields

$$
c(x) \leqq \frac{3}{2}+O\left(\frac{\log \log \log x}{\log \log x}\right)
$$

Combining (3.19) with (3.22) completes the proof of Lemma 2.

We now consider a lemma which will enable us to extend the conclusion of Theorem 2 to all integers of the form $2^{n}-1$.

LEMMA 3. If $x=2^{N}-1$, then there exists an even integer $M \geqq N$ such that $M-N \leqq \sqrt{N} / \log N, M / 2=\sum_{i=1}^{r} 2^{a_{1}}-1$ with $a_{r} / a_{1} \geqq 1 / 2-\epsilon$ and

$$
A_{2}(x)=\frac{A_{2}\left(2^{M}-1\right)}{2^{M-N}}+O(x)
$$

where

$$
\epsilon=\epsilon(x)=\frac{\log \log \log x}{\log \log x+\log \log 2}
$$

Proof. Let $N=\sum_{i=1}^{l} 2^{a_{1}}, a_{1}>a_{2}>\cdots>a_{1}$. Define $n$ by $n+1=$ $\sum_{1} 2^{c_{*}}$; where $\left\{c_{l}\right\}$ runs over all integer values in the interval $\left[1,(1 / 2) a_{1}(1-2 \epsilon)+1\right]$ not equal to any of the $a_{1}$ 's. If no such $c$ 's exist, let $n=0$ if $N$ is even, $n=1$ if $N$ is odd. Let $M=N+n$. Clearly $n=M-N \ll 2^{(1 / 2) a_{1}(1-2 \epsilon)} \ll N^{1 / 2-\epsilon} \ll \sqrt{N} / \log N$ and only (3.23) requires further analysis.

As before, $A_{2}\left(2^{M}-1\right)=\Sigma_{s \leqq M}\left(\begin{array}{c}M \\ s\end{array}\right) \alpha(s)$.

We rewrite this as

$$
A_{2}\left(2^{M}-1\right)=s_{1}+s_{2}
$$

where 


$$
\begin{gathered}
s_{1}=2^{n} \sum_{s}\left(\begin{array}{c}
N \\
s
\end{array}\right) \alpha(s)=2^{M-N} A_{2}\left(2^{N}-1\right) \\
s_{2}=\sum_{s}\left\{\left(\begin{array}{c}
M \\
s
\end{array}\right)-2^{n}\left(\begin{array}{c}
N \\
2
\end{array}\right)\right\} \alpha(s) .
\end{gathered}
$$

We bound $s_{2}$ from above by writing

$$
s_{2} \ll \log M \sum_{s}\left|\left(\begin{array}{c}
M \\
s
\end{array}\right)-2^{N}\left(\begin{array}{c}
N \\
2
\end{array}\right)\right| .
$$

But

$$
\begin{aligned}
\sum_{s}\left|\left(\begin{array}{c}
M \\
s
\end{array}\right)-2^{N}\left(\begin{array}{l}
N \\
s
\end{array}\right)\right| & =\sum_{s}\left|\sum_{q}\left(\begin{array}{c}
N \\
s-q
\end{array}\right)\left(\begin{array}{l}
n \\
q
\end{array}\right)-\left(\begin{array}{l}
N \\
s
\end{array}\right)\left(\begin{array}{l}
n \\
q
\end{array}\right)\right| \\
& \leqq \sum_{q}\left(\begin{array}{l}
n \\
q
\end{array}\right) \sum_{s}\left|\left(\begin{array}{c}
N \\
s-q
\end{array}\right)-\left(\begin{array}{l}
N \\
s
\end{array}\right)\right| \\
& \leqq \sum_{q}\left(\begin{array}{l}
n \\
q
\end{array}\right) \cdot 2 q \max \left(\begin{array}{l}
N \\
s
\end{array}\right) \ll n \cdot 2^{n} \cdot 2^{N} / \sqrt{N} \\
& \ll \frac{\sqrt{N}}{\log N} \cdot 2^{n} \cdot 2^{N} / \sqrt{N}=\frac{2^{N+n}}{\log N}
\end{aligned}
$$

so $s_{2} \ll 2^{N+n} \ll 2^{n} x=2^{M-N} x$ and

$$
A_{2}\left(2^{M}-1\right)=2^{M-N}\left\{A_{2}(x)+O(x)\right\},
$$

proving the lemma.

Corollary 1. If $x=2^{N}-1$, then

$$
\frac{1}{2}+O\left(\frac{\log \log \log x}{\log \log x}\right) \leqq c(x) \leqq \frac{3}{2}+O\left(\frac{\log \log \log x}{\log \log x}\right)
$$

Proof. Find an $M$ as in Lemma 3 so that

$$
A_{2}(x)=\frac{A_{2}\left(2^{M}-1\right)}{2^{M-N}}+O(x)
$$

Applying Lemma 2 to $2^{M}-1$ immediately yields this result.

LEMMA 4. Let $x=\sum_{i=1}^{r} 2^{s_{i}}, s_{1}>s_{2}>\cdots>s_{r .}$ Then 


$$
A_{2}(x)=\sum_{i=1}^{r} A_{2}\left(2^{s_{i}}-1\right)+O(x \log \log x / \sqrt{\log x})
$$

Proof.

$$
A_{2}(x)=\sum_{i=1}^{r} \sum_{n<2^{s_{1}}} \alpha_{2}\left(\sum_{j=1}^{i-1} 2^{s_{1}}+n\right)
$$

Since $\alpha\left(\sum_{j=1}^{i-1} 2^{s,}+n\right)=\alpha(n)+i-1$ we obtain

$$
A_{2}(x)=\sum_{i=1}^{r} \sum_{n<2^{s_{1}}} \alpha(\alpha(n)+i-1)
$$

Letting $E_{i}=\Sigma_{n<2^{s},}\{\alpha(\alpha(n)+i-1)-\alpha(\alpha(n))\}$, we obtain

$$
A_{2}(x)=\sum_{i=1}^{r} A_{2}\left(2^{s_{t}}-1\right)+\sum_{i=2}^{r} E_{i}
$$

We now must merely show that $\sum_{i=2}^{r} E_{i}=O(x \log \log x / \sqrt{\log x})$. Rewrite

$$
\begin{aligned}
& E_{i}=\sum_{\substack { l \leq s_{1} \\
\begin{subarray}{c}{n<2, i-1 \\
\alpha(n)=l{ l \leq s _ { 1 } \\
\begin{subarray} { c } { n < 2 , i - 1 \\
\alpha ( n ) = l } }\end{subarray}}\{\alpha(l+i-1)-\alpha(l)\} \\
& =\sum_{l}\left(\begin{array}{l}
s_{i} \\
l
\end{array}\right)\{\alpha(l+i-1)-\alpha(l)\} .
\end{aligned}
$$

Summing by parts,

$$
E_{i}=\sum_{l} \alpha(l)\left\{\left(\begin{array}{c}
s_{t} \\
l-i+1
\end{array}\right)-\left(\begin{array}{l}
s_{l} \\
l
\end{array}\right)\right\}
$$

Since $\alpha(l)=O\left(\log \left(s_{t}+i\right)\right)$ and

$$
\sum_{l}\left|\left(\begin{array}{c}
s_{l} \\
l-i+1
\end{array}\right)-\left(\begin{array}{c}
s_{i} \\
l
\end{array}\right)\right|=O\left(i\left(\begin{array}{c}
s_{i} \\
{\left[s_{i}\right] / 2}
\end{array}\right)\right)=O\left(i \frac{2^{s_{i}}}{\sqrt{s_{l}}}\right)
$$

we obtain

$$
E_{\imath} \ll i \cdot \log \left(s_{\imath}+i\right) 2^{s_{i}} / \sqrt{s_{i}} .
$$




$$
\sum_{i=2}^{r} E_{i} \ll \sum_{i=2}^{r} i \log \left(s_{i}+i\right) 2^{s_{i}} / \sqrt{s_{i}}
$$

Since $s_{t} \leqq s_{1}-i+1$ and $s_{t}+i \ll \log x$, and writing $s=s_{1}$, we obtain

$$
\sum_{i=2}^{\prime} E_{i} \ll \log \log x \sum_{i=1}^{r} i 2^{s-i} / \sqrt{s-i}
$$

Now

$$
\sum_{i \leq s / 2} i 2^{s-i} / \sqrt{s-i} \ll \frac{2^{s}}{\sqrt{s}} \sum \frac{i}{2^{i}} \ll 2^{s} / \sqrt{s}
$$

while

$$
\sum_{i>s / 2} i 2^{s-i} / \sqrt{s-i} \ll \sum_{i>s / 2} i 2^{s-i} \ll s \cdot 2^{s / 2} \ll \frac{2^{s}}{\sqrt{s}} .
$$

Since $2^{s}=O(x)$ and $s=\log x / \log 2+O(1)$, we obtain

$$
\sum_{i=2}^{r} E_{i}=O(x \log \log x / \sqrt{\log x})
$$

completing the proof of Lemma 4 .

We can now easily prove Theorem 2 .

Proof of Theorem 2. Let $x=\sum_{t=1}^{r} 2^{s_{i}}$. By Lemma 4,

$$
A_{2}(x)=\sum_{i=1}^{r} A_{2}\left(2^{s_{t}}-1\right)+O(x)
$$

Corollary 1 implies that

$$
A_{2}\left(2^{s_{i}}-1\right) \leqq \frac{3}{2} \frac{2^{s_{i}} \log \log x}{2 \log 2}+O\left(2^{s_{i}} \log \log \log x\right),
$$

so that

$$
A_{2}(x) \leqq \frac{3}{2} \sum_{i=1}^{r}\left(\frac{2^{s_{i}} \log \log x}{2 \log 2}+O\left(2^{s_{i}} \log \log \log x\right)\right)+O(x)
$$




$$
A_{2}(x) \leqq \frac{3}{2} \frac{x \log \log x}{2 \log 2}+O(x \log \log \log x)
$$

which is equivalent to

$$
c(x) \leqq \frac{3}{2}+O\left(\frac{\log \log \log x}{\log \log x}\right)
$$

We now obtain a lower bound. Again using Corollary 1, we obtain

$$
A_{2}(x) \geqq \frac{1}{2} \sum_{i=1}^{r}\left(\frac{2^{s_{i}} \log \log 2^{s_{i}}}{2 \log 2}+O\left(2^{s_{1}} \log \log \log x\right)\right)+O(x)
$$

and hence

$$
A_{2}(x) \geqq \frac{1}{2} \sum_{i=1}^{r} \frac{2^{s_{i}} \log \log 2^{s_{i}}}{2 \log 2}+O(x \log \log \log x) .
$$

Since $\log \log 2^{s_{1}}=\log \log x+O(1)$ if $s_{\imath} \geqq s_{1} / 2$, we obtain

$$
A_{2}(x) \geqq \frac{1}{2} \sum_{s_{1} \geq s_{1} / 2} \frac{2^{s_{4}} \log \log x}{2 \log 2}+O(x \log \log \log x) .
$$

But $\Sigma_{s_{1} \geq s_{1} / 2} 2^{s_{x}}=x+O\left(2^{s_{1} / 2}\right)=x+O(\sqrt{x})$ yielding

$$
A_{2}(x) \geqq \frac{1}{2} \frac{x \log \log x}{2 \log 2}+O(x \log \log \log x)
$$

which implies

$$
c(x) \geqq \frac{1}{2}+O\left(\frac{\log \log \log x}{\log \log x}\right)
$$

completing the proof of Theorem 2 .

\section{REFERENCES}

1. R. Bellman and H. N. Shapiro, On a problem in additive number theory, Annals of Math., 49 (1948), 333-340.

2. L. Mirsky, A theorem of representations of integers in the scale of $r$, Scripta Math., 15 (1949), 11-12. 
3. I. Shiokawa, On a problem in additive number theory, Math. J. Okayama University, 16 (1973-74), 167-176.

4. S. C. Tang, An improvement and generalization of Bellman-Shapiro's theorem on a problem in additive number theory, Proc. Amer. Math. Soc., 12 (1963), 199-204.

Received September 14, 1976 and in revised form May 16, 1977. Work by the first author was partially supported by The University of Connecticut Faculty Summer Fellowship, 1975, and work by the second author was partially supported by the National Science Foundation, Grant NSFMPS-75-08545.

THE UNIVERSITY OF CONNECTICUT

WATERBURY, CT

AND

COLUMBIA UNIVERSITY

NEW YORK, NY 10027 



\section{PACIFIC JOURNAL OF MATHEMATICS}

\section{EDITORS}

RICHARD ARENS (Managing Editor)

University of California

Los Angeles, CA 90024

R. A. BeAumont

University of Washington

Seattle, WA 98105

C. C. MOORE

University of California

Berkeley, CA 94720
J. DUGUNDJI

Department of Mathematics

University of Southern California

Los Angeles, CA 90007

R. FINN AND J. MILGRAM

Stanford University

Stanford, CA 94305

\section{ASSOCIATE EDITORS}
E. F. BECKENBACH
B. H. NEUMANN
F. WOLF
K. YoshidA

\section{SUPPORTING INSTITUTIONS}

UNIVERSITY OF BRITISH COLUMBIA

UNIVERSITY OF SOUTHERN CALIFORNIA

CALIFORNIA INSTITUTE OF TECHNOLOGY

STANFORD UNIVERSITY

UNIVERSITY OF CALIFORNIA

UNIVERSITY OF HAWAII

MONTANA STATE UNIVERSITY

UNIVERSITY OF TOKYO

UNIVERSITY OF NEVADA

UNIVERSITY OF UTAH

NEW MEXICO STATE UNIVERSITY

OREGON STATE UNIVERSITY

UNIVERSITY OF OREGON

OSAKA UNIVERSITY

WASHINGTON STATE UNIVERSITY

UNIVERSITY OF WASHINGTON

AMERICAN MATHEMATICAL SOCIETY

The Supporting Institutions listed above contribute to the cost of publication of this Journal, but they are not owners or publishers and have no responsibility for its contents or policies.

Mathematical papers intended for publication in the Pacific Journal of Mathematics should be in typed form or offset-reproduced (not dittoed), double spaced with large margins. Underline Greek letters in red, German in green, and script in blue. The first paragraph or two must be capable of being used separately as a synopsis of the entire paper. Items of the bibliography should not be cited there unless absolutely necessary, in which case they must be identified by author and Journal, rather than by item number. Manuscripts, in duplicate, may be sent to any one of the four editors. Please classify according to the scheme of Math. Reviews, Index to Vol. 39. All other communications should be addressed to the managing editor, or Elaine Barth, University of California, Los Angeles, California, 90024.

100 reprints are provided free for each article, only if page charges have been substantially paid. Additional copies may be obtained at cost in multiples of 50 .

The Pacific Journal of Mathematics is issued monthly as of January 1966. Regular subscription rate: $\$ 72.00$ a year (6 Vols., 12 issues). Special rate: $\$ 36.00$ a year to individual members of supporting institutions.

Subscriptions, orders for numbers issued in the last three calendar years, and changes of address should be sent to Pacific Journal of Mathematics, 103 Highland Boulevard, Berkeley, California, 94708.

PUBLISHED BY PACIFIC JOURNAL OF MATHEMATICS, A NON-PROFIT CORPORATION

Printed at Jerusalem Academic Press, POB 2390, Jerusalem, Israel. 


\section{Pacific Journal of Mathematics}

\section{Vol. 75, No. $2 \quad$ October, 1978}

Susan Jane Zimmerman Andima and W. J. Thron, Order-induced

topological properties ................................... 297

Gregory Wade Bell, Cohomology of degree 1 and 2 of the Suzuki groups . . 319

Richard Body and Roy Rene Douglas, Rational homotopy and unique

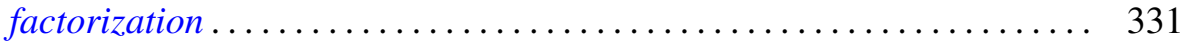

Frank Lewis Capobianco, Fixed sets of involutions ................. 339

L. Carlitz, Some theorems on generalized Dedekind-Rademacher sums .... 347

Mary Rodriguez Embry and Alan Leslie Lambert, The structure of a special class of weighted translation semigroups .....................

Steve Ferry, Strongly regular mappings with compact ANR fibers are

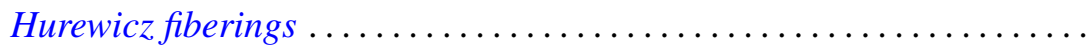

Ivan Filippenko and Marvin David Marcus, On the unitary invariance of the

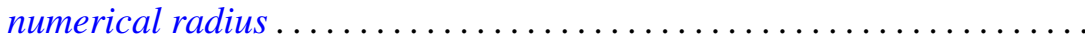

$\mathrm{H}$. Groemer, On the extension of additive functionals on classes of convex

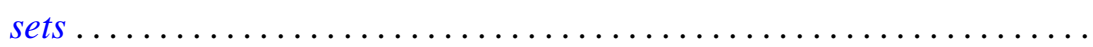

Rita Hall, On the cohomology of Kuga's fiber variety ............... 411

H. B. Hamilton, Congruences on $\mathrm{N}$-semigroups ................. 423

Manfred Herrmann and Rolf Schmidt, Regular sequences and lifting

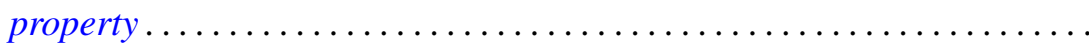

James Edgar Keesling, Decompositions of the Stone-Čech compactification which are shape equivalences .....................

Michael Jay Klass and Lawrence Edward Myers, On stopping rules and the expected supremum of $S_{n} / T_{n}$

Ronald Charles Linton, $\lambda$-large subgroups of $C_{\lambda}$-groups

William Owen Murray, IV and L. Bruce Treybig, Triangulations with the free cell property ............................

Louis Jackson Ratliff, Jr., Polynomial rings and $H_{i}$-local rings ...

Michael Rich, On alternate rings and their attached Jordan rings....

Gary Sampson and H. Tuy, Fourier transforms and their Lipschitz

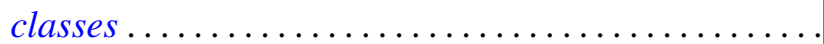

Helga Schirmer, Effluent and noneffluent fixed points on dendrites ...

Daniel Byron Shapiro, Intersections of the space of skew-symmetric maps

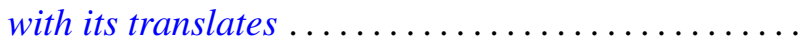

Edwin Spanier, Tautness for Alexander-Spanier cohomology ...

Alan Stein and Ivan Ernest Stux, A mean value theorem for binary digits ...

Franklin D. Tall, Normal subspaces of the density topology . .

William Yslas Vélez, Prime ideal decomposition in $F\left(\mu^{1 / p}\right) \ldots$ 